\title{
Wireless Sensor Network energy balance routing based on ant colony
}

\section{algorithm}

\author{
Wang Xin $^{1, a, *}$, Shi Ying ${ }^{1, b}$ \\ ${ }^{1}$ Information technology teaching and Management Center, Jilin Agricultural \\ University, Changchun 130118, China \\ jarryxin@126.com, b30166933@qq.com
}

Keywords: ant colony algorithm, Wireless Sensor Network, life cycle, energy balance

\begin{abstract}
The traditional limitations of ant colony algorithm used in Wireless Sensor Networks was studied sufficiently and improved infectively in this paper. The node residual energy pheromone is considered as update factor and distance heuristic probability function is also included in this method to improved wireless sensor routing algorithm based on ant colony algorithm. Simulation experiments was carried out through the establishment of wireless sensor network model, its result shows that the proposed algorithm converges faster than traditional method, guarantees nodes balance of energy consumption in the networks. In additional, the network life cycle was increased by $10 \%$ compare with traditional method.
\end{abstract}

\section{Introduction}

Wireless sensor networks consist of plenty nodes with information sensing and data communication function which can collect and process information in monitoring area, then send to observers by self-organizing multi hop network. Different from the traditional network routing with time delay and data transmission rate, the sensor nodes are powered by micro battery. Therefore, the sensor network routing protocol pays more attention to the energy consumption of nodes. In routing design for Sensor Networks, it is necessary to minimize the energy consumption. Otherwise, global balance of energy consumption is also important. Inspired by behaviour of ants in nature, ant colony algorithm was proposed for the first time in 1991 by Colorni[1] to solve a series of optimization problems. The routing problem has some characteristics such as distributed computing, random dynamic, asynchronous state which is matching with ant colony algorithm, so ant colony algorithm can provide a novel way to solve the problem of network routing. 


\section{Relevant Research}

There are already lots of research about ant colony routing algorithm for wireless networks. Literature[2] proposed AntNet routing protocol first, domestic and foreign researchers put forward the corresponding improvement measures subsequently. Zhang[3] proposed that heuristic factor constructed by nodes distance can make evaluation for time delay, total energy consumption and energy efficiency of uniform distributed sensor networks. Tu[4] found that the ant colony routing algorithm formulated by total energy for message passing in routing minimizes network energy consumption, but it cannot guarantee energy balance. Ming-Hua L [5] improved the pheromone update process, due to nodes energy distribution is considered in the routing, the energy balance of the network was optimized. The algorithm in [6] reduces the packet type by ant routing table, when the node pheromone is lower than a threshold, it enters the sleep mode to save energy. But it is easy to form a local optimum, which is not conducive to the energy balance of the whole network. For efficiency of ant search, Luo Z et al [7] proposed a ACO algorithm based on energy and location awareness which obtains location information of the node, neighbour node and sink node by location technology, but it is not enough for specific quantitative analysis in energy consumption.

\section{Ant colony algorithm}

Ant colony algorithm is a probabilistic algorithm for finding an optimal path in a graph that was initially applied to solve the traveling salesman problem (TSP). Subsequently, it becomes to be a heuristic algorithm for solving combinatorial optimization problems. Implementation of ant colony algorithm includes two basic processes of path building and pheromone updating.

The path construction rule is to determine the probability of the next hop, shown as $(1)$

where is pheromone, is heuristic factor, , is the distance from node $i$ to node $j$, is neighbour node set that ant $\mathrm{k}$ can get in node $\mathrm{i}$, and are parameters which show importance of pheromone and heuristic factors. For each pass, the shorter is, the greater the probability is.

The ants leave pheromone on the path and the pheromone will evaporate over time. After all ants have completed a search, the pheromone of each pass will be updated, shown as (2),

where is pheromone release, is pheromone decay coefficient, .

and are two important parameters in the design of routing algorithm, heuristic factors determines exploring ability of ants, and the pheromone release affects the convergence speed. Relative details will be discussed in the following parts. 


\section{Ant colony routing algorithm design}

\section{1 factor analysis}

It is known that information probability transfer mechanism based on distance heuristic is adopted in the first iteration in classic ant colony algorithm. Therefore, the next step is determined by the transition probability function, which depends on pheromone. However, in the initial stage the pheromone is not obvious and contributes little to the path choice. So, an adjusted distance heuristic probability function was adopted in this paper to calculate the transition probability and improve initial search performance.

In the first iteration, heuristic information probability function based on distance was used to calculate the transition probability to improve the initial search performance.

(3)

where is the transition probability of ant $k$ from sensor node $i$ to node $j$, is neighboring nodes set that has not been visited by ant $\mathrm{k}$, is the distance between node $\mathrm{j}$ and target node. , , are all constant.

In order to advance converge rate, an improvement based on (1) shown as (4)

is shown as (5),

(5)

\section{2 heuristic factor construction}

There are lots of nodes in wireless sensor networks and the nodes are always densely distributed, the sensor nodes can not maintain the global information. The topology of the network changes frequently because of dead nods based on the energy consumption. the design was adjusted that the residual energy of nodes and hops are used as the factors of pheromone updating shown as (6),

(6)

where and are average energy and minimum energy of all nodes, is initial energy. the average energy of all nodes on the path to describe the routing cost can guarantee the global energy balance.

The valve of node $\mathrm{m}$ is , denotes residual energy. The higher the value of the residual energy, the higher the value of the node is, the residual energy of the node is reduced and the value of the node is increased. The cost of forwarding data is (7),

is the energy consumed by forwarding packets. The larger the packet, the smaller the residual energy of the node, and the higher the forwarding cost.

Thus, the factors of pheromone updating are increased to energy, distance and the cost above, so we got the pheromone updating viable as (8),

(8)

is the sum of the residual energy of the nodes ant k passed, is numbers of hops, is coefficient. 


\section{Simulation results and analysis}

There are 200 sensor nodes randomly distributed in the area $200 * 200$ sensing area, the sink node is located on the region boundary location $(0,0)$, and a random node in the upper right corner is chosen as source node, shown as Fig.1. The parameters of the simulation experiment are shown in Tab.1 and experiment run by MATLAB7.8.0.

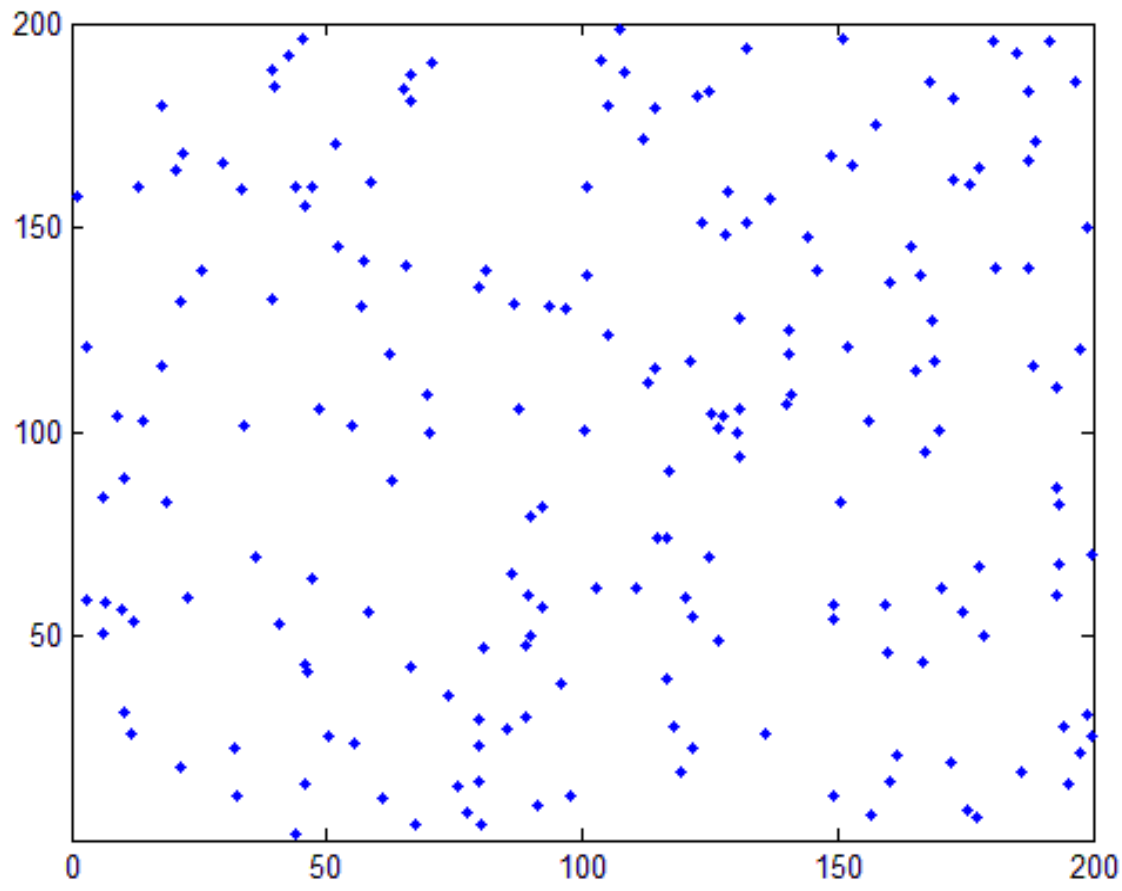

Fig.1 Distribution of sensor nodes in simulation

Tab.1 Simulation experiment parameters

\begin{tabular}{|l|l|l|l|}
\hline Parameters & Value & Parameters & value \\
\hline & 0.2 & & 0.1 \\
\hline & 1.5 & & 0.1 \\
\hline & 1 & & 1 \\
\hline Packet length & 1000 bit & Initial energy of nodes & $1 \mathrm{~J}$ \\
\hline
\end{tabular}

The average number of hops is shown as Fig. 2 compared between the improved ant colony algorithm in this paper and the basic ant colony algorithm. With the increase of iterations, the average hops of two algorithms both reduced, but the improved routing algorithm occupied a lower degree network resources, its network bandwidth consumption is smaller. That is help to alleviate network congestion and data packets can be transmitted in the network more quickly. Simulation results show that the routing algorithm based on improved ant colony algorithm is more efficient. 


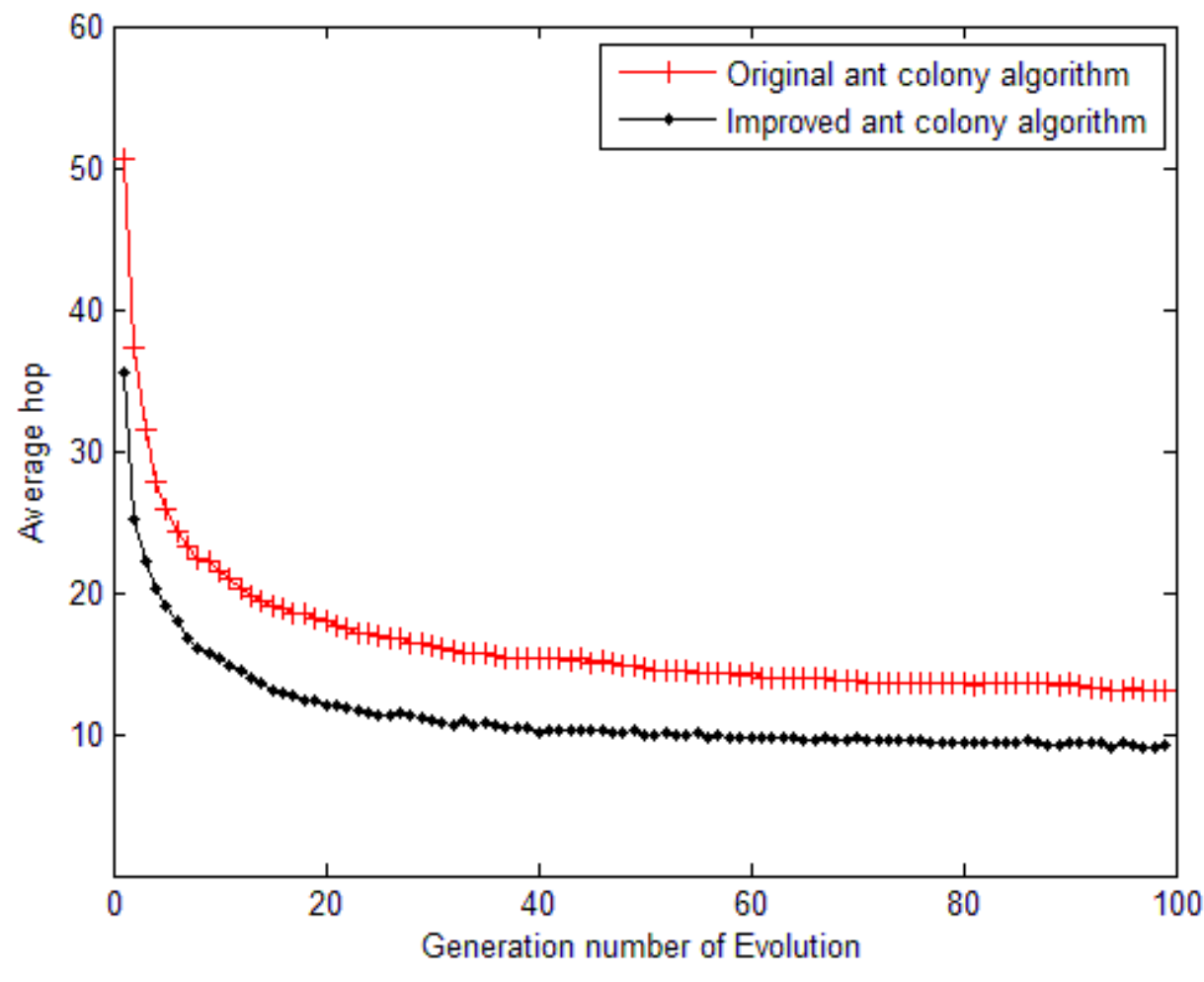

Fig. 2 The average number of hops comparison

Comparative analysis of network energy consumption is shown as Fig. 3. As the number of iterations increases, the total energy consumed by each iteration is reduced. the total energy consumption is $0.245 \mathrm{~J}$ in classic method, and $0.204 \mathrm{~J}$ in our method. By comparison we can also see that the improved ant colony algorithm has better convergence. 


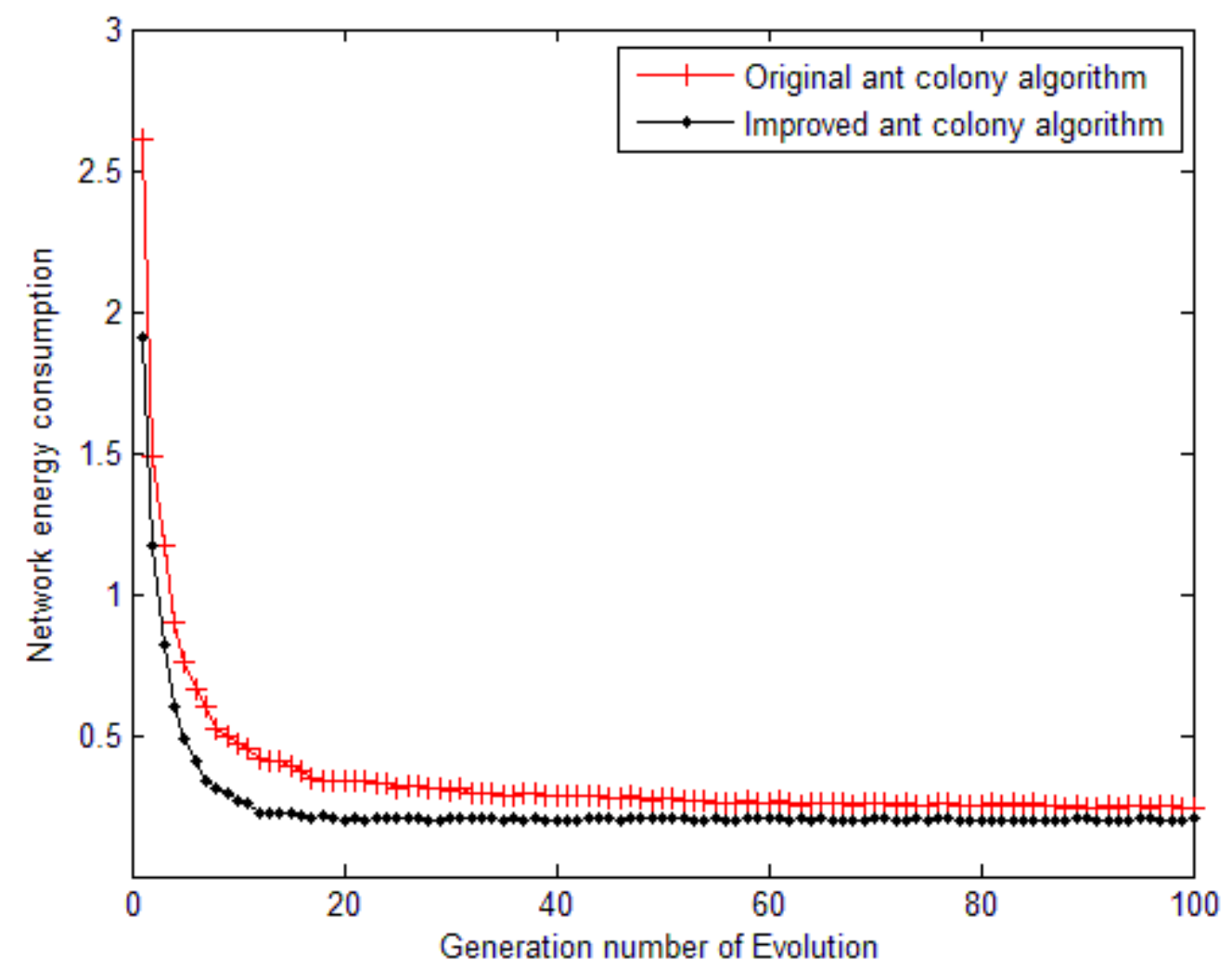

Fig.3. Comparative of network energy consumption

\section{Conclusion}

The convergence of the basic ant colony algorithm is slow and easy to fall into local optimal value. Due to that, we improved the algorithm and applied to routing algorithm in Wireless Sensor Networks. The performance of the improved ant colony algorithm and the original ant colony algorithm is simulated and compared. The simulation results show that the improved algorithm has better performance in average number of hops and lower energy consumption.

\section{- Acknowledgements}

"Research on routing protocol for Lifetime Optimization in Wireless Sensor Networks”, Jilin Agricultural University research foundation in 2015, 2015045.

\section{- References}

[1] Colorni A,Dorigo M, Maniezzo V. Distributed optimization by ant colonies [C], European Conference on Artificial Life,1991. Cambridge: MIT Press,1991: 134-142.

[2] Di Caro G,Dorigo M. AntNet:distributed stigmergetic control for communication networks 
[J].Journal of Artificial Intelligence Research,1998,9(1): 317-365.

[3] Zhang Y,Kuhn L D, Fromherz M P J. Improvements on ant routing for sensor networks[C]\|International Work- shop on Ant Colony Optimization and Swarm Intelligence, 2004. Brussels: Springer Verlag,2004: 154-165.

[4] Tu Z,Wang Q, Shen Y. Optimal mobile agent routing for data fusion in distributed sensor networks using improved ant colony algorithm[C]\|IEEE International Conference on Instrumentation and Measurement Technology,2008. Victoria: IEEE,2008: 155-159.

[5] Ming-Hua L, Hua Z, Guang S. Energy aware routing algorithm for wireless sensor network based on ant colony principle[J]. Journal of Convergence Information Technology, 2012.

[6] Cobo L, Quintero A, Pierre S. Ant-based routing for wireless multimedia sensor networks using multiple QoS metrics[J]. Computer Networks, 2010, 54(17):2991-3010.

[7] Luo Z, Lu L, Xie J, et al. An ant colony optimization-based trustful routing algorithm for wireless sensor networks[C]// International Conference on Computer Science and Network Technology. IEEE, 2016:1128-1131. 